\title{
Name codes and features in the discrimination of letter forms
}

\author{
YEI-YU YEH and CHARLES W. ERIKSEN \\ University of Illinois, Champaign, Illinois
}

\begin{abstract}
This research addressed the role of name and physical codes in visual discriminations and also tested the appropriateness of discrete stage models of information processing as opposed to continuous flow models. One group of subjects was required to discriminate the uppercase letters $A$ and $E$ from the set $G$ and $Q$. Another group discriminated between the lowercase counterparts, set $a$ and $e$ and set $g$ and $q$. RT was the dependent variable. A response competition paradigm was used in which the target letter was flanked by "noise" letters that were either response compatible or response incompatible. The noise letters could also be either in the same case as the target or in the alternate case. Thus, it was possible for the noise letters to be either physically identical to the target or identical in name only, and their features could be similar to the target in either one of the response sets. Although physical features of the stimuli played the major role in discrimination, name codes were found to facilitate performance when target and noise shared the same name. Feature similarity between noise letters and targets of the opposite response set produced interference, supporting a continuous flow conception of visual information processing.
\end{abstract}

The research reported below addresses two issues of current concern in cognitive psychology. The first pertains to the role of name and physical codes in visual discriminations. The second concerns the appropriateness of discrete stage models of information processing as opposed to models or conceptions that conceive processing more as a cascade or continuous flow. We will address first the issue of physical and name codes. Then, after presentation of the experimental design, we will point out how it relates to and tests the question of discrete versus continuous processing models.

The role that name or phonetic codes play in the discrimination among visual forms has become theoretically important in several contexts. The problem had received interest back in the 1950s when the proposition that name codes contribute to the distinctiveness of forms and thus improve their discriminability was tested. Although a definitive answer was not achieved by research at that time, the evidence suggested that name codes contributed little to discriminability (Hake \& C. W. Eriksen, 1956; Prentice, 1958).

More recent research has focused on how name codes enter into information processing and the types of tasks that activate them. Egeth, Blecker, and Kamlet (1969) found that the verbal, or name, component was relatively unimportant in matching single-stimulus attributes on a classification task, and Flowers and Dutch (1976) concluded that the relative efficiency of name and physical codes

This research was supported by Public Health Service Research Career Program Award K6-MH-22014 to the second author and by U.S. Public Health Service Research Grant MH-01206. The authors' mailing address is: Department of Psychology, University of Illinois, 603 East Daniel, Champaign, IL 61820. on perceptual classifications was largely dependent upon the memory requirements of the task.

Much of the recent interest and theorizing about name codes has arisen from experiments that employed a matching task with letter stimuli. On these tasks, the subjects can be asked to match on the basis of physical identity or on the basis of name identity. A consistent finding has been that even when the subjects are matching on the basis of "name same," the match is faster if the stimuli are also physically identical. This name-physical disparity, as Proctor (1981) has termed it, has been interpreted as due to differences in latencies for making matches on the basis of a physical code as opposed to the name code. Proctor has further implicated name codes as the basis for the disparity in latency of same and different judgments. Typically, "same" judgments are made more rapidly than judgments of "different." In Proctor's general theory of matching behavior, this same-different disparity is attributed to competition and resulting inhibition between the two name codes that are activated when the stimulus pair is different as opposed to the single name code that is activated when the stimuli are identical or nominally the same.

Posner (1978) and Posner and Snyder (1975) have proposed that when a familiar form is presented the input activates independently the physical code and the phonetic (name) code. The evidence for this independent activation of name and physical codes, as reviewed by Posner (1978), consists primarily of experimental manipulations that affect the speed with which one code or the other is available without concurrently affecting the speed of activation of the other code.

While there is a good deal of face validity to the assumption that stimuli that are only nominally the same are 
matched on the basis of name codes, Boles (1981) and Boles and Eveland (1983) have presented impressive evidence that indicates that name codes are not typically involved in same-different matches, even when the matches are at the nominal level. Boles (1981) has pointed out that matches made on the basis of physical representations or codes and matches based on name codes should show lateralization effects between the hemispheres. His research failed to find any consistent lateralization effects. Boles and Eveland (1983), in a series of experiments, failed not only to find evidence of name codes involved in matching tasks, but also to replicate two experiments (Dainoff \& Haber, 1978; Thorson, Hochhaus, \& Stanners, 1976) that had provided much of the evidence for the involvement of name codes in same-different matches. Instead of name codes, Boles and Eveland found evidence for generation of visual or physical codes even when the to-be-matched stimuli were presented successively with relatively long $(2 \mathrm{sec})$ intervening intervals.

There is another level at which name codes could influence discrimination in matching task behavior other than at a conscious level. The semantic priming experiments that show the facilitation effects on mental processing time due to semantic and associative relatedness (Fischler, 1977; Meyer \& Schvaneveldt, 1976; and Neely, $1976,1977)$ are generally interpreted as automatic or unconscious effects. Tanenhaus, Flanigan, and Seidenberg (1980) propose that an input activates all of its codesphonological, orthographic, and semantic. The evidence they present shows that a prime activates along orthographic and phonological dimensions in both visual and auditory word recognition. This automatic activation of all the codes for an input offers the potential for facilitation and inhibition effects in the processing of the input. This automatic activation of a name code may facilitate the generation of the physical code, and the simultaneous activation of two or more name codes may inhibit and delay the generation or the development of the physical code. In this latter instance, there would be a basis for Proctor's theory of same-different disparity, even if the subject's match was not made on the basis of name codes.

The present experiment used upper- and lowercase letters from the English alphabet and a response competition paradigm developed by B. A. Eriksen and C. W. Eriksen (1974). One group of subjects was required to discriminate the target set $G$ and $Q$ from the set $A$ and $E$ by moving a response lever in one direction if the presented letter was a $G$ or a $Q$ and in the opposite direction for an $A$ or an $E$. A second group of subjects performed the same task, with the targets being the lowercase counterparts $\mathrm{a}$ and $\mathrm{e}$ and $\mathrm{g}$ and $\mathrm{q}$. The response competition paradigm consisted of surrounding these target letters with noise letters that called for either the same-direction lever response or a response in the opposite direction. Both groups of subjects were informed that the target letter would always occur immediately above the fixaton point and were told to ignore any other letters that appeared in the visual field. With the exception of control trials, the target letter was flanked by a noise letter on each side.
The two noise letters were always identical to each other. The noise letters flanking the target could be (1) identical in name and physical characteristics to the target, e.g., A A A; (2) name-same as the target, e.g., a A a; (3) response compatible with the target, e.g., E A E or e A e; or (4) response incompatible with the target, e.g., GA G or g A g. ${ }^{1}$

From prior work with the response competition paradigm (B. A. Eriksen \& C. W. Eriksen, 1974; C. W. Eriksen \& B. A. Eriksen, 1979; C. W. Eriksen \& Hoffman, 1973; C. W. Eriksen \& Schultz, 1979), we know that when the noise is response incompatible with the target, reaction time to the target is appreciably increased. Noise letters that are identical to the target have little effect upon target latency as compared to a control no-noise condition, whereas response-compatible noise letters tend to slightly increase reaction time to the target. These results have been interpreted as showing, first, that the subject cannot attend exclusively to a position in space and ignore irrelevant stimuli that fall on effective retinal areas; second, that the noise or irrelevant letters are processed essentially simultaneously with the target letter to the point of incipient response activation; and, third, that the activation of the competing response by a noise letter from the opposite target set inhibits, and thus slows, the correct discriminated reaction time.

In the present experimental design, our choice of letter stimuli permits varying noise-target relationship, not only in terms of response compatibility, but also in terms of feature and name relationship. The noise letters could be physically identical to the target, e.g., A A A, or identical in name only with feature similarity to targets of the opposite response set, e.g., a A a. Thus, we could vary the noise feature similarity to the target or to the opposite target set while holding constant the name relationship of the target and the noise. Unfortunately, the design does not permit us to hold feature similarity constant while varying name, but this limitation is largely overcome by varying feature similarity through the use of upper- and lowercase target sets, e.g., A E and GQ and $a$ e and $g q$. By systematically using upper- and lowercase noise with upper- and lowercase target sets across responsecompatibility conditions, the design should provide information on the relative contribution of name codes versus features in target recognition and choice reaction time.

As we stated earlier, this experiment also addresses the issue of continuous versus discrete-stage models in information processing. Both implicitly and explicitly discrete stage models have had an important influence on cognitive theory and experimentation. Perhaps the most prominent example is Sternberg's (1969) elegant set of experiments, which used the memory scan paradigm. The additive factors method that he developed provides a powerful analytic tool for partitioning reaction time into the underlying mental operations or processes. However, the power of this analytic tool is critically dependent upon the assumption of discrete stages.

In their elemental form, discrete-stage models are distinguished by the assumption that the various mental oper- 
ations that occur from the moment a stimulus impinges upon a sense organ to the point at which response occurs are discrete in the sense that each operation is completed before the next operation begins. Each stage completes its processing of the input before it passes the results of its processing on to the next mental operation or stage (output buffering) or the next stage stores the output from the preceding stage (input buffering) until it is complete before beginning its operaton or processing.

There have been objections to discrete-stage modeling on the basis of neurophysiological evidence (Vaughan \& Ritter, 1973) as well as on theoretical and empirical bases (C. W. Eriksen \& Schultz, 1979; Grice, Nullmeyer, \& Spiker, 1982; McClelland, 1979; Miller, 1982a, 1982b, 1983; Norman \& Bobrow, 1975; Turvey, 1973). Alternative models have been proposed which can be characterized as continuous. These differ in the important conception that the output of any mental operation to other operations or processes is continuous. That is, the ongoing results of one operation can be fed to the next operation before the first one has completed its analysis of the input.

Many years back, Guthrie (1952, p. 143) criticized Tolman's cognitive theory of learning on the grounds that it left the rat buried in thought. A similar criticism can be applied today to many of our cognitive models. Too little attention is directed toward how responses are activated or evoked.

Continuous models have attempted to rectify this oversight, and most of the empirical evidence supporting continuous flow processing has come from an examination of subtle effects upon responses. Miller (1982a, 1982b, 1983) has found important evidence for continuous processing in terms of facilitating effects via response preparation using multidimensional stimuli that vary in discriminability of the component dimensions. Grice and his colleagues (Grice, Nullmeyer, \& Spiker, 1977, 1982) have developed a quantitative measure of the growth of associative strength leading to response evocation and, further, have shown the effect of competing stimuli upon the growth of this associative strength (Grice, Canham, \& Schafer, 1982). C. W. Eriksen and Schultz (1979) proposed their continuous flow model of visual information processing primarily to account for the effects of response competition and the differential rate with which different stimulus dimensions are processed.

The experiment outlined above provides a critical test of the continuous flow model proposed by $\mathrm{C}$. W. Eriksen and Schultz as well as the variable criterion model of Grice and his colleagues. Although variable criterion theory was developed as a general model of human reaction time and the continuous flow model arose from observations on response competition in visual discrimination tasks, both models share important assumptions in common. Both assume that information in the form of a percept develops gradually over time in the visual system, and that response priming begins as the percept is developing. At early stages of percept development, a wide range of responses receive initial priming, but as the information continues to accumulate in the percept, the priming flow is restricted increasingly to those responses that still remain viable alternatives. A response is evoked when the priming in that response reaches a preset criterion. The continuous flow model further assumes that the speed with which the response is executed depends upon the amount of priming in competing responses. The greater the priming in competing responses, the greater the inhibition of the correct response and the longer its latency in execution.

Neither the continuous flow model nor variable criterion theory allow for a decision stage in choice reaction time from which responses are activated or initiated. Instead, the subject is assumed to adopt a criterion for the task. This criterion may vary somewhat over trials as the subject adjusts his performance to the standard of accuracy expected. The criterion the subject adopts on a trial determines the level of prime that a response has to reach before the response is evoked.

Both models assume that relevant responses receive a priming flow as features associated with the stimulli for these responses become available or discernible in the developing percept. In the present experiment, noise letters are presented along with the target letter. If these noise letters have features that are associated with the response opposite to that required for the target, both models would expect that these features, as they became delineated in the developing percept, would cause some priming to the competing response. Consequently, the latency of response to the target would be greater then than it would be if the noise letter had few or no features associated with the opposite response target.

In the present experiment, the uppercase target group provides a test of the continuous flow model and also variable criterion theory. Consider the condition in which the target is from the response set $A$ and $E$ and the noise is name identical and same case, e.g., A A A, versus name identical but alternate case, e.g., a $\mathrm{A}$ a. The response set is $\mathrm{G}$ and $\mathrm{Q}$, either of which, if presented as a target, would lead to a lever response in the opposite direction. In both cases, A A A and a A a, the target is flanked by a responsecompatible letter if the name label is used, irrespective of whether the noise is upper- or lowercase. But, in the instance in which the lowercase letter is the noise, the name is compatible but the features of the lowercase letter are quite similar to the other response set, $G$ and $Q$. The latency of response to the target $A$ would be expected to be greater if the competing response appropriate to $G$ or $Q$ was primed by the feature similarity that a and e share with the curved features of $G$ and $Q$ than it would if it was flanked by As. The case of the noise should have less effect when $G$ and $Q$ are the targets and the noise letters have the same name, e.g., G G G and g G g, than when the target set is A and E. The lowercase form of $g$ and $q$ has low feature similarity to $A$ and $E$. This should result in a low level of priming for the competing response associated with $\mathrm{A}$ and $\mathrm{E}$. Thus, the continuous flow model 
would require that the lowercase name-identical noise should produce a greater relative increase in latency for $A$ and $E$ targets than for $G$ and $Q$.

This outcome would also be strong evidence against discrete-stage theory. Discrete stages can accommodate the results of response competition where the latency of response to the target is increased if a noise letter of the opposite target set is present in the field. Both the target and noise letter could be processed in discrete stages, but in parallel, and on some trials the noise letter might complete processing first and the decision maker partially activate the inappropriate response. Alternatively, both noise and target could complete the processing simultaneously and be available to the decision maker, which again partially activates the inappropriate response as it vacillates between the target and noise letter. However, it is difficult to conceive how a strictly discrete-stage model could accommodate the outcome if reaction time to the target was appreciably greater between A A A and a A a than it was for $\mathrm{G} \mathrm{G} \mathrm{G}$ and $\mathrm{g} \mathrm{G} g$ or, alternatively, E E E and e E e versus $Q Q Q$ and $q Q q$. If perceptual or input processing is completed before the outcome is available to a decision maker, then it should not make any difference whether the target is A or E or G or Q. Either upper- or lowercase noise is identified as the respective letter, and whether or not it has featural similarity to the opposite response target should not prime a competing response.

Similar predictions hold for the condition in which the noise is response compatible, e.g., E A E or G Q G. Here the noise letter would prime and activate the same response as the target, but when the target was an $\mathrm{A}$ or an $\mathrm{E}$, shifting to the lowercase form (e $\mathrm{Ae}$ or a $\mathrm{E}$ a) would again present features that should tend to prime the response appropriate to the $G Q$ targets. On the other hand, when the target is a $G$ or $Q$ lowercase gs and qs as noise would have less priming effect on the competing response appropriate to $A$ and $E$ due to the lack of feature similarity in $g$ and $q$ to the uppercase $A$ and $E$ targets.

When a noise letter is response incompatible, e.g., G A G, the case of the noise letters would tend to have the opposite effect. With the A E targets, $g$ and $q$ would be expected to activate the competing response on the basis of both features and name codes. For $G$ and $Q$ targets, the use of $a$ and $e$ as the incompatible noise would reduce the activation of the competing lever response since $a$ and e are dissimilar on features to the opposite response targets $\mathrm{A}$ and $\mathrm{E}$.

\section{METHOD}

\section{Design}

The experiment was a mixed four-way factorial. One group of subjects was assigned uppercase letters as targets and the other subject group was assigned the lowercase counterparts as the targets. The remaining variables were within-subject effects. They were: (1) targets (A and $E$ or $a$ and $e$ versus $G$ and $Q$ or $g$ and q); (2) noise type [noise with the same name as the target, noise that had the same lever response as the target (compatible), or incompatible noise where the noise letter had a response opposite to that required by the target]; and (3) noise case (the noise could either be in the same case as the target or in the alternate case).
In Figure 1, these different treatment combinations are shown along with the displays that were presented to the subjects. In addition to the above treatments, a control, or no-noise, condition in which the target letter was presented alone in the display was counterbalanced with the other treatments.

Originally, four subjects were run with the upper- and four with the lowercase targets. Approximately a year and a half later, the experiment was replicated with an additional four subjects in each of the two groups. Because the replication produced essentially identical results, the data are presented as the results of a single experiment.

\section{Subjects}

Sixteen University of Illinois graduate students served as paid volunteers. All had normal or corrected-to-normal vision. Eight were in the lowercase target group and eight in the uppercase group.

\section{Apparatus and Stimuli}

The stimuli were presented in two channels of a Scientific Prototype three-channel tachistoscope illuminated with F4 T5/CWX bulbs. Viewing was binocular. Luminance of the white background for all fields was set at $9 \mathrm{fL}$. Reaction time was recorded to the nearest millisecond on a Hunter Model 1522 digital Klockounter.

Stimulus displays were constructed on white vinyl cards using Futura Bold 20-point black type for all uppercase letters, 28-point type for the lowercase $\mathrm{a}$ and $\mathrm{e}$, and 24-point type for the lowercase $\mathrm{g}$ and $\mathrm{q}$. These different type point sizes were used to provide an approximately equal size for all letters, although some variations in stroke width existed as a result. All letters subtended about $.25^{\circ}$ in width and height. The displays were constructed so that the target letter would always occur $.25^{\circ}$ of angle above a fixation cross that appeared in the adapting field. The two noise letters that were presented with the target letter on a trial were identical to each other and flanked the target letter by a separation of $.5^{\circ}$ of visual angle at the viewing distance of 48 in

\begin{tabular}{|c|c|c|c|c|}
\hline \multirow{3}{*}{$\begin{array}{l}\text { Noise Conditions - } \\
\text { Same Case } \\
\text { Name } \\
\text { identical }\end{array}$} & \multicolumn{4}{|c|}{ Torgets } \\
\hline & \multicolumn{2}{|c|}{ Upper Case } & \multicolumn{2}{|c|}{ Lower case } \\
\hline & $\begin{array}{l}\text { A A A } \\
\text { G G G }\end{array}$ & $\begin{array}{l}\text { E E E } \\
\text { QQQ }\end{array}$ & $\begin{array}{l}0 \mathbf{a} \\
9 g \mathrm{~g}\end{array}$ & $\begin{array}{l}\text { eqe } \\
\text { q q q }\end{array}$ \\
\hline $\begin{array}{l}\text { Response } \\
\text { compatible }\end{array}$ & $\begin{array}{l}\text { E A E } \\
\mathbf{A} \mathbf{Q}\end{array}$ & $\begin{array}{l}A E A \\
G Q G\end{array}$ & $\begin{array}{l}\text { eqe } \\
999\end{array}$ & $\begin{array}{l}090 \\
999\end{array}$ \\
\hline $\begin{array}{l}\text { Response } \\
\text { incompatible }\end{array}$ & $\begin{array}{l}\text { GAG } \\
\text { QA } \\
A G A \\
\text { E G E }\end{array}$ & $\begin{array}{l}\text { GEG } \\
Q \text { E Q } \\
\text { A QA } \\
\text { E QE }\end{array}$ & $\begin{array}{l}909 \\
q \propto 9 \\
090 \\
e 9 e\end{array}$ & $\begin{array}{l}g e g \\
q e q \\
q q a \\
e q e\end{array}$ \\
\hline \multicolumn{5}{|l|}{ Noise Conditions - } \\
\hline \multicolumn{5}{|l|}{ Opposite Case } \\
\hline $\begin{array}{l}\text { Name } \\
\text { identical }\end{array}$ & $\begin{array}{l}0 A G \\
9 G 9\end{array}$ & $\begin{array}{l}\text { e E e } \\
\mathbf{q} \mathbf{q}\end{array}$ & $\begin{array}{l}\mathbf{A} \propto \mathbf{A} \\
\mathbf{G} \mathbf{G}\end{array}$ & $\begin{array}{l}\text { E } \\
\mathbf{a} \mathbf{a}\end{array}$ \\
\hline $\begin{array}{l}\text { Response } \\
\text { compatible }\end{array}$ & $\begin{array}{l}\text { 수 } \\
\text { gG }\end{array}$ & $\begin{array}{l}0 \text { E } \\
9 Q 9\end{array}$ & $\begin{array}{l}\text { E E } \\
\text { Q } 9 Q\end{array}$ & $\begin{array}{l}A \in A \\
G 9 G\end{array}$ \\
\hline $\begin{array}{l}\text { Response } \\
\text { incompatible }\end{array}$ & $\begin{array}{l}9 A 9 \\
9 A 9 \\
0 G Q \\
\text { eGe }\end{array}$ & $\begin{array}{l}9 \text { E } \\
q \text { E } \\
\text { qQa } \\
\text { eqe }\end{array}$ & $\begin{array}{l}\mathbf{G} \mathbf{G} \\
\mathbf{Q} \mathbf{Q} \\
\mathbf{A} \mathbf{A} \mathbf{A} \\
\mathbf{E} \mathbf{E}\end{array}$ & $\begin{array}{l}\text { GeG } \\
\text { Qe } \\
\text { A } 9 \text { A } \\
\text { E } 9 \text { E }\end{array}$ \\
\hline
\end{tabular}

Figure 1. The visual displays presented to the subjects in the different conditions of the experiment. The center letter in all cases was the target, and the flanking letters, the noise. Center letter position was designated by a fixation point prior to display onset, and the subjects were instructed to ignore the flanking noise letters. 


\section{Procedure}

Subjects in both the upper- and lowercase target groups served in two individual sessions. A session consisted of 30 practice trials and four blocks of 56 trials (eight trials under each of the six noise conditions and eight trials under the no-noise control). Within a trial block, each target letter occurred with equal frequency in a pseudorandom order.

The subjects were instructed to hold a handswitch in the left hand for initiating the stimulus presentation and to place the right hand on a handrest with thumb and forefinger holding a response lever. They were told to press the button with the left hand only when the fixation cross was clearly in focus. They were told that the target letter would always appear immediately above the fixation cross and were instructed to ignore other letters that might occur, simultaneously with the target, in the flanking positions. The subjects were asked to respond as quickly and as accurately as possible. Half of the subjects in each group were told to move the lever to the left for an A or an $\mathrm{E}$ and to the right for $\mathrm{a} G$ or a $\mathrm{Q}$. The other half of the subjects were given the reverse instructions.

When the subject initiated a trial by pressing the button with his or her left hand, the fixation field went off and the stimulus display appeared for $1 \mathrm{sec}$. When the display terminated, it was replaced by the fixation field. Two lights, visible only to the experimenter, indicated the direction of the subject's lever response. Any trial on which the subject moved the lever in the wrong direction or in both directions was rerun late in the session. Error data were recorded. After every trial, the subject was provided with feedback regarding his or her accuracy and reaction time.

\section{RESULTS}

Mean latencies of the correct responses to the target under each of the noise conditions are shown in Figure 2 for the subject group using uppercase targets and in Figure 3 for the group using lowercase targets. ${ }^{2}$ These data (the no-noise condition was excluded) were analyzed in a five-way ANOVA (case of the target letter, target set,

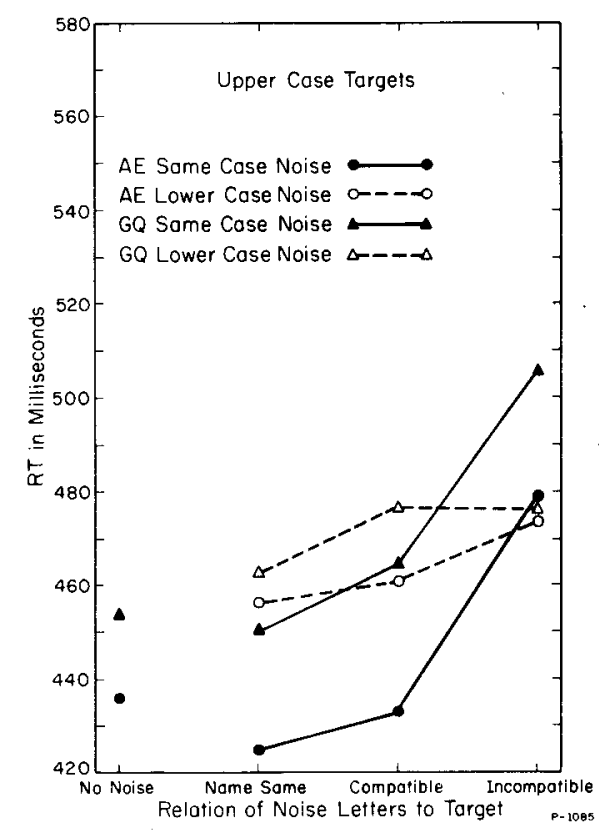

Figure 2. Mean reaction time of correct responses as a function of the relationship between the target and noise letter for the subjects using uppercase targets. The two symbols on the left side of the graph represent performance under the no-noise condition.

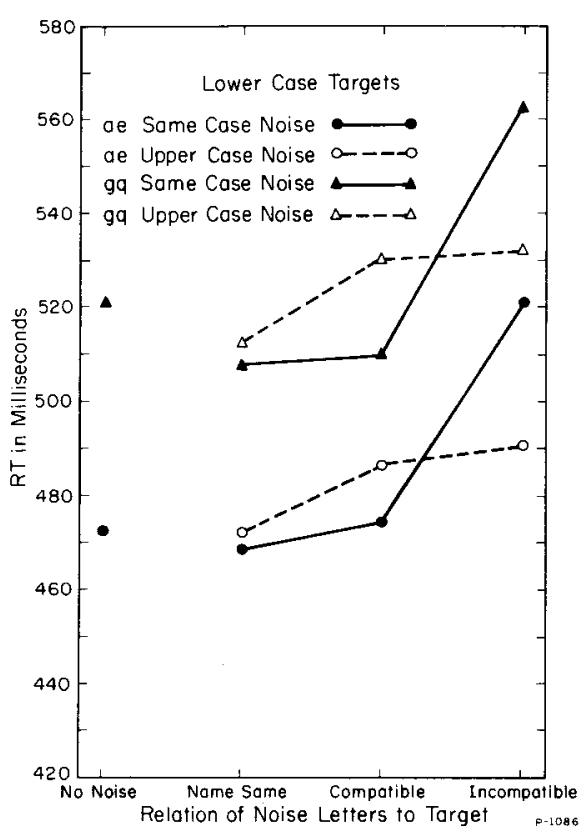

Figure 3. Mean reaction time of correct responses as a function of the relationship between the target and noise letters for subjects using lowercase targets. The two symbols on the left side of the graph represent performance under the no-noise condition.

same- versus different-case noise letters, noise compatibility, and subjects). The uppercase targets were responded to faster than the lower case $[F(1,14)=5.71, p<.05]$. The AE (a e) targets were responded to faster than the GQ (g q) targets $[F(1,14)=15.61, \mathrm{p}<.005]$. The response compatibility of the noise was a highly significant effect $[F(2,28)=54.04, p<.0001]$. Reaction time was significantly longer $(\mathrm{p}<.01)$ in the incompatiblenoise condition than in the compatible condition, in which, in turn, it was significantly longer than it was in the namesame condition, as determined by post hoc comparisons. The interaction between response compatibility and the case of the noise was significant $[F(2,28)=30.21$, $\mathrm{p}<.0001$ ], as was also the interaction of targets $\times$ the case of the noise $\times$ the case of the targets $[F(1,14)=5.05$, $\mathrm{p}<.05]$.

noise $\times$ the case of the targets $[F(1,14)=5.05, p<.05]$.

Previous research using the response competition paradigm typically has found that when the target letter is flanked by noise letters that are identical to the target, RT is essentially the same as when the target is presented alone without accompanying noise letters (no-noise control). When the noise letters have the same experimentally assigned response as the target letter, there is a small, but statistically reliable, increase in RT to the target. A pronounced increase in RT, on the other hand, is obtained when the noise letters are members of the other target set, calling for a different response. The results obtained with noise letters in the same case as the target in the present experiment are a replication of these previous findings on noise compatibility and target reaction time. The results demonstrate that the response competition effect is operat- 
ing in the present experimental arrangement and, more importantly, provide the base line for evaluating the effect of the other experimental variables.

A major variable is the effect of noise letters when they are in a different case from the target sets. By changing the case, we maintain constant the name-code relations between the noise letters and the targets but vary the featural similarity, not only between the noise and the targets, but also between the noise and the letters in the other target set. The data in Figures 2 and 3 indicate that both the name code and the features are involved in the subject's discriminated reaction time to letters. The most clear evidence of the role of name codes is found in a comparison of alternate-case noise in the name-same and compatible-noise conditions. For all four target sets, RT to the target letter is less if the flanking noise letters have the same name as the target than if they have the name of the other member in that target set, i.e., response compatible. Averaged across the four target sets, the effect is significant by planned comparisons beyond the .05 level.

Although the above comparison establishes that name codes are entering into this discrimination task, the physical features of the stimuli independent of name code appear to have an even more pronounced effect. For both the name-same and the compatible-noise conditions, changing the noise to the alternate case signifiantly increases reaction time even though the names have remained constant. A further indication of the importance of the physical features of the stimuli is seen in the incompatiblenoise condition. Here, although the name of the noise is still that of the stimuli calling for the opposite response, the interference produced on target RT is appreciably less than if these incompatible noise stimuli are the same case as the target. Across upper- and lowercase targets and target sets, the increase in reaction time for incompatible noise of the alternate case is not significantly larger than it is in the compatible-noise condition. By contrast, when noise and target are the same case, the incompatible-noise condition is appreciably slower than the compatible noise condition $(\mathrm{p}<.001)$.

The results obtained for the AE in the incompatiblenoise condition is atypical when the lowercase incompatible noise is employed. With the other three target sets ( $G Q$, a e, and g q), alternate-case noise in the incompatible condition results in appreciably less interference on target RT than is obtained when the noise is the same case as the target. But, with the A E targets, the incompatible noise in the form of $\mathrm{g}$ and $\mathrm{q}$ produces nearly as much impairment in RT as when the noise is the same case as the target. This difference for the A E target set is expected because under these circumstances not only has the name of the noise remained constant, but a good deal of the featural similarity is present in terms of the curved features of the $\mathbf{G}$ and the $Q$. Thus, in this instance, we have both name codes and features acting together to produce interference via competing responses.

As we pointed out in the introduction, the uppercase target group provides a test of the continuous flow model in visual information processing. The target set $\mathrm{A} E$ differs from the set GQ in that the former has features consisting of straight lines and angles whereas the latter consists predominantly of curved features. Thus, the discrimination between the two target sets should be easier than the discrimination between the lowercase targets. But the lowercase counterparts of these uppercase target sets are quite similar in that they consist of curved features.

In discriminating $A E$ from $G Q$, curved features can be presumed to activate a lever response in one direction whereas straight lines and angles should prime a response in the opposite direction. In the name-same and compatible-noise conditions, in which the noise is the same case as the target, the noise letters for both the A E and the GQ target sets are consistent with the target features, that is, they would tend to prime the same response as they become discernible in the developing percept. However, in these two noise conditions, when the lowercase form of the noise is used, the targets A E are flanked by noise letters that have curved features highly similar to the opposite target set $\mathrm{GQ}$, e.g., a A a or e A e. In terms of the continuous flow model, these curved features would prime the opposite lever movement response. Thus, conflict and inhibition should occur. For the target set $\mathrm{GQ}$ on the other hand, shifting to the lowercase versions of noise, namely $\mathrm{g} \mathrm{q}$, maintains the curved features in both the noise and the target. Since little similarity to the opposite response set $\mathrm{AE}$ is present in these displays, only minimal activation or priming of the competing response would be anticipated. In other words, there should be a greater difference between same- and alternate-case noise for the A E targets than there should be for the $G Q$ targets. For the incompatible-noise condition, the substitution of a $e$ for $A \mathrm{E}$ as noise for $\mathrm{GQ}$ targets should reduce the amount of interfering competition, but with the A E targets, substitution of $\mathrm{g} \mathrm{q}$ for $\mathrm{G} Q$ should have little effect, since both features and name code should prime the competing lever response.

Examination of the data in Figure 1 for these uppercase targets shows that the data conform with these predictions. The significance of the effects was evaluated by performing three-way ANOVAs (target set, same vs. alternate case of the noise letter, and subjects) on the data for each of the three noise conditions separately. In all three analyses, the predicted interaction between target set and same- versus alternate-case noise was significant beyond the $.05 \mathrm{lev}$ el. For the name same and compatible noise conditions, shifting to lowercase noise produced a significantly greater increase in latencies for the $A E$ target set than for the $G Q$. For the incompatible noise condition, the use of the alternate-case noise reduced interference for the GQ.

\section{GENERAL DISCUSSION}

The results of this experiment support the conclusion that both the name code and the physical features of the stimulus, as represented in the percept, are involved in the discrimination between letter forms. As would be ex- 
pected, the evidence indicates that the physical features play the the dominant role in discrimination. The data show a pronounced effect when the case of the noise letters is different from that of the target, particularly with the uppercase targets A E and GQ. Here the results are in accord with the prediction of the continuous flow model and are compatible with the variable criterion theory of Grice et al. (1982). Reaction times to the target in the name-same and compatible-noise conditions are appreciably increased if the case of the noise letters is changed so that they have high feature similarity to the other target set. This is the expected outcome if features, as they become available in the developing percept, prime responses that are appropriate to these features. Conversely, if changing the noise to the alternate case decreases the similarity to the alternate target set, they inhibit reaction time to a target less than if the noise consisted of the alternate targets.

As we noted in the introduction, discrete stage models can account for response competition effects by assuming that on some trials the noise letter completes processing or becomes available to the decision stage before the target. This results in a partial activation of the inappropriate response, which would then inhibit the correct response when the true target became available. But, if this explanation were correct, we would not expect feature similarity between target and incompatible noise to produce the pronounced effect that it does. If the response stage is isolated or not involved until the stimulus is processed to identification, either on a physical or name-code basis, there is no reason to expect a $\mathrm{A}$ a to produce more competition than $\mathrm{g} \mathrm{G} \mathrm{g}$ or e E e more than $\mathrm{q} \mathrm{Gq}$. But, if responses are being primed as features become discernible in the developing percept, the differential performance obtained in the two conditions is what would be expected. Our data and the results of Miller, 1982a, 1982b, 1983) seem to require models that recognize that responses are involved early in stimulus processing as opposed to models that envisage a decision stage and then response activation.

In the present experiment, the clearest evidence for the importance of name codes in the discrimination between letter stimuli comes from a comparison between the namesame condition and the compatible-noise condition when the noise is in the alternate case to that of the target. In all four instances, reaction time to the target letter flanked by name-same letters is less than that obtained in the compatible-noise condition. Although these data show that name codes do enter into simple visual discriminations of letters, the means by which they do so is far from clear. We can speculate as to several possible mechanisms.

When a significant variable has been found using averaged data, we can never be certain whether it contributes a constant amount on every trial or occurs in an "all or none" fashion and is present on only a certain proportion of the trials. In well-practiced subjects, the discrimination may be made on the basis of the physical features or characteristics of the stimuli on most of the trials, but on occasional trials the name may occur and be used as a basis for discrimination. C. W. Eriksen $(1956,1957)$ has presented evidence to show that when multiple responses exist for a stimulus, they are activated a least partially independently. More recently, Posner (1978) and Posner and Snyder (1975) have proposed that when a familiar form is presented, the input activates independently the physical code and the phonetic or name code. This concept of independent activation of name and physical codes would provide a means by which the name code could influence the discrimination on a certain proportion of trials.

C. W. Eriksen and O'Hara (1982) note that even if the average latency for activation of the physical code is shorter than the average latency for activation of the name code, the name code would be available before the physical code on a certain proportion of trials as long as the two distributions of latency overlap. If a large number of trials were run in the experiment, the effect of a name code would be observed. In terms of the continuous flow model or of variable criterion theory, we could assume that the subject's response criterion was based upon the physical code. On those trials on which the name code became available or was activated before the percept had reached the criterion based on the physical code, the name code could interfere with the match if it was incompatible with the targets. If the name code of a noise letter was the same as the target, facilitation, or at the least no interference, would be obtained.

This explanation for the present results cannot be ruled out, but it should be noted that if this were the mechanism by which name codes were influencing the discrimination, one would anticipate a greater interference effect in the incompatible noise condition. The proportion of times that the name code was available before the percept developed to the response criterion should be the same in the compatible, incompatible, and name-same conditions. Thus, on those trials on which the name was available first, one would expect a pronounced interference effect in the incompatible-noise condition. The data show, however, that with alternate-case noise reaction time to the target is not significantly or appreciably greater than that for the compatible noise condition (with the predicted exception for $\mathrm{A}$ and $\mathrm{E}$ targets).

Further evidence against a parallel activation of name code and the lever-movement response is found in the work of Boles and Eveland (1983). The work of those investigators casts considerable doubt on the common interpretation that nominal matches of letter pairs in same-different discrimination are based upon name codes. Not only do Boles and Eveland present evidence based upon original experiments of their own, but in essential replications of Dainoff and Haber (1978) and Thorson et al. (1976), they fail to confirm the results of these latter investigators. The experiments of Dainoff and Haber and or Thorson et al. had both reported that effects of acoustic similarity of extraneous letter stimulation have interfered with nominal matches of letter pairs. As such, these findings had constituted important evidence for name code involvement in simultaneous and successive same-different discrimination in those matches based on "name same." 
Although Boles and Eveland (1983) employed matching tasks, their failure to find name code involvement even in nominal matches would certainly suggest that the subjects in our experiment were not discriminating between target letter sets on the basis of name. There would be much more reason for name codes to be involved in nominal letter matches than in a simple visual discrimination.

Although the Boles and Eveland experiments suggest that matches are not made on the basis of the name code, this does not necessarily require that name codes do not enter into the processing and development of the physical codes. In addition to the data of the present experiment, there is other evidence that name codes are involved. $C$. W. Eriksen and O'Hara (1982) used a letter-matching task in conjunction with the response competition paradigm. They presented, simultaneously, target letter pairs to be matched on nominal identity. Response competition was introduced by presenting, at irrelevant positions in the display, noise letters that either matched or did not match the target or were similar to one target letter but not the other. They also employed letter-like nonsense forms as noise stimuli. At the completion of the first half of the experiment, subjects were given a learning session in which they learned to associate letter names to these nonsense forms . Following the learning session, the subjects completed two more sessions of same-different judgments with the various noise stimuli. By comparing the interfering effect of the nonsense forms before and after learning, it was evident that the names the subjects had learned to associate with these nonsense forms were producing interference effects comparable to their real letter counterparts (although of less magnitude).

But how can the name code affect discrimination or matching if it is not occurring as the conscious counterpart of the discrimination on at least a certain percentage of the trials? Semantic priming experiments have shown that the effects of antecedent stimuli can be quite pervasive as well as subtle. The work of Tanenhaus et al. (1980) has indicated that these priming effects can occur at both the phonetic and the orthographic level. Priming effects are quite compatible with the continuous flow model of information processing ( C. W. Eriksen \& Schultz, 1979). Features in the developing percept are assumed to activate name codes which, since they are partially activated, could in turn prime or facilitate the development of the physical code or the percept. If the noise and target letter activate different name codes, even though both codes are for members of the same target set, this dual activation of name codes could result in mutual inhibition. This would slow the processing of both and cancel out the facilitation effect that is obtained when target and noise letter are activating the same name code.

The operation of such a process could account for the failure to find a significant difference between the compatible and the incompatible noise conditions with the alternate-case noise letters in the present experiment. As noted above, if the name code of the noise letter was fully activated, greater inhibitory effects upon the response to the target letter should have been obtained in the incompatible-noise condition. In terms of the above speculation, the name codes are never fully activated but exert their influence solely upon the speed of processing of the physical code. Under these circumstances, their facilitory or inhibitory effect is dependent solely upon whether they are partially activating the same name code or different name codes. Thus, it should be immaterial whether the noise letter is the other member of the target set or a member of the opposite-response target set. In both instances, noise and target letters are activating different name codes, with the result that facilitation in processing the target's physical code is lacking.

This explanation would require that reaction time to target letters be faster when the noise is physically identical to the target than in the control condition in which the target is presented alone. The evidence on this point is unclear. In the present experiment, the physically identical noise did produce faster RTs than the no-noise control, but the difference was not significant. This has been a typical finding in many of our experiments. However, Flowers and Wilcox (1982) have obtained suggestive evidence of facilitation. Part of the problem revolves around the choice of an appropriate comparison value for determining whether facilitation occurred. In a comparison of a control condition in which the target occurs alone and a condition in which the target occurs flanked by a noise letter that is identical, any facilitation effect that the latter condition provides may be masked. Not only is there the presence of the potentially facilitating stimuli in the second condition, but it also differs from the first in that there are three separate stimuli in the field. Grice, Canham, and Schafer (1982) have shown that the presence of an irrelevant stimulus in choice reaction time tasks acts to delay the growth of associative strength. Furthermore, C. W. Eriksen and Hoffman (1972) found that such innocuous stimuli as black disks in the visual field impaired reaction time to clearly designated target letters. Also, Eriksen, Hamlin, and Day (cited in C. W. Eriksen \& Schultz, 1978) found that the presentation of geometric forms and patches of color delayed reaction time to target letters. In all instances, the target was clearly differentiated and segregated from this accompanying noise. Most recently, Treisman, Kahneman, and Burkell (1983) have reported very similar results. Thus, it is quite possible that the failure to find a clear facilitation effect when the noise is physically identical with the target could be due to the increased processing capacity demanded by the additional stimuli in the effective visual field, which counteracts or offsets any gain from target redundancy.

\section{REFERENCES}

Boles, D. B. (1981). Variability in letter matching asymmetry. Perception \& Psychophysics, 29, 285-288.

Boles, D. B., \& EVELAND, D. C. (1983). Visual and phonetic codes and the process of generation in letter matchings. Journal of Experimental Psychology: Human Perception and Performance, 9, 657-674.

DAINOFF, M. J., \& HABER, R. N. (1978). Effect of acoustic confusability 
on levels of information processing. Canadian Journal of Psychology, 24, $98-188$

Egeth, H. E., Blecker, D. L., \& KAmlet, A. S. (1969). Verbal interference in a perceptual comparison task. Perception \& Psychophysics, 6, 355-356.

Eriksen, B. A., \& EriksEN, C. W. (1974). Effects of noise letters upon the identification of a target letter in a nonsearch task. Perception \& Psychophysics, 16, 143-149.

Eriksen, C. W. (1956). Subception: Fact or artifact? Psychological Review, 63, 74-80.

ERIKSEN, C. W. (1957). Prediction from and interaction among multiple concurrent discriminative responses. Journal of Experimental Psychology, 53, 132-138.

ERIKSEN, C. W. \& ERIKSEN, B. A. (1979). Target redundancy in visual search: Do repetitions of the target within the display impair processing? Perception \& Psychophysics, 26, 195-205.

ERIKSEN, C. W. , \& HofFMAN, J. E. (1972). Temporal and spatial characteristics of selective encoding from visual displays. Perception \& Psychophysics, 12, (2B), 201-204.

ERIKSEN, C. W. , \& Hoffman, J. E. (1973). The extent of processing of noise elements during selective encoding from visual displays. Perception \& Psychophysics, 14, 155-160.

ERIKSEN, C. W., \& O'HARA, W. P. (1982). Are nominal same-different matches slower due to differences in level of processing or to response competition? Perception \& Psychophysics, 32, 335-344.

ERIKSEN, C. W., SCHULTZ, D. W. (1978). Temporal factors in visual information processing. In J. Requin (Ed.), Attention and performance VII. New York: Academic Press.

ERIKSEN, C. W., \& SchULTZ, D. W. (1979). Information processing and visual search: The continuous flow conception and experimental results. Perception \& Psychophysics, 29, 249-263.

Fischler, I. (1977). Associative facilitation without expectancy in a lexical decision task. Journal of Experimental Psychology: Human Perception \& Performance, $3,18-26$.

FLowERS, J. H., \& DuTCH, S. (1976). The use of visual and name codes in scanning and classifying colors. Memory \& Cognition, 4, 384-390.

Flowers, J. H., \& Wilcox, N. (1982). The effect of flanking context on visual classification: The joint contribution of interactions at different processing levels. Perception \& Psychophysics, 32, 581-592.

Grice, G. R., Canham, L., SChafer, C. (1982). Development of associative and perceptual interference. Perception \& Psychophysics, 32, 375-387.

Grice, G. R., Nullmeyer, R., \& Spiker, V. A. (1977). Application on variable criterion theory to choice reaction time. Perception \& Psychophysics, 22, 431-449.

Grice, G. R., Nulimeyer, R., \& SPIKer, V. A. (1982). Human reaction time: Toward a general theory. Journal of Experimental Psychology: General, 11, 135-153.

GUTHRIE, E. R. (1952). The psychology of learning. New York: Harper \& Row.

HAKE, H. W., \& ERIKSEN, C. W. (1956). Role of response variables in recognition and identification of complex visual forms. Journal of Experimental Psychology, 52, 235-243.

MCCLELLAND, J. L. (1979). On the time relations of mental processes: An examination of systems of processes in cascade. Psychological Review, 86, 287-330.

MeYer, D., \& SChVANeveldT, R. W. (1976). Meaning, memory structure, and mental processes. Science, 192, 27-33.
Miller, J. (1982a). Discrete versus continuous stage models of human information processing: In search of partial output. Journal of Experimental Psychology: Human Perception and Performance, 8, 273-296.

MilLER, J. (1982b). Effects of noise letters on decisions: Discrete or continuous flow of information? Perception \& Psychophysics, 31, 227-236.

MILLER, J. (1983). Can response preparation begin before stimulus recognition finishes? Journal of Experimental Psychology: Human Perception and Performance, 9, 161-182.

NeELY, J. H. (1976). Semantic priming and retrieval from lexical memory: Evidence for facilitory and inhibitory processes. Memory \& Cognition, 4, 648-654.

NeELY, J. H. (1977). Semantic priming and retrieval from lexical memory: Roles of inhibitionless spreading activation and limited-capacity attention. Journal of Experimental Psychology: General, 106, 226-254.

Norman, D., \& BoBRow, D. (1975). On data-limited and resource-limited processes. Cognitive Psychology, 7, 44-64.

PoSnER, M. K. (1978). Chronometric explorations of the mind. Hillsdale, NJ: Erlbaum.

POSNER, M. K., \& SNYDER, C. R. R. (1975). Facilitation and inhibition in the processing of signals. In P. M. A. Rabbitt \& S. Dornic (Eds.), Attention and performance $V$. New York: Academic Press.

Prentice, W. C. H. (1958). Perception. Annual Review of Psychology, 9, 1-18.

Proctor, R. W. (1981). A unified theory for matching task phenomena. Psychological Review, 88, 291-326.

STERNBERG, S. (1969). The discovery of processing stages: Extensions of Donder's method. Acta Psychologika, 30, 276-315.

Tanenhaus, M. K., Flanigan, H. P., Seidenberg, M. S. (1980). Orthographic and phonological activation in auditory and visual word recognition. Memory \& Cognition, 8, 513-520.

Thorson, G., Hochhaus, L., \& Stanners, R. F. (1976). Temporal changes in visual and acoustic codes in a letter-matching task. Perception \& Psychophysics, 19, 346-348.

Treisman, A., Kahneman, D., \& Burkell, J. (1983). Perceptual objects and the cost of filtering. Perception \& Psychophysics, 33, 527-532.

TURVEY, M. (1973). On peripheral and central processes in vision: Inferences from an information processing analysis of masking with pattern stimuli. Psychological Review, 80, 1-52.

VAughan, H. G., JR., \& RitreR, W. (1973). Physiologic approaches to the analysis of attention and performance. In S. Kornblum (Ed.), Attention and performance, IV. New York: Academic Press.

\section{NOTES}

1. Although the examples of displays given in the text show a size difference between upper- and lowercase letters, the size of the letters in the experimental displays were the same, as can be seen in Figure 1.

2 . The presentation of results will deal only with latencies because error rates were quite low $(1 \%$ to $13 \%$, with a mean of $5.5 \%)$ and reflected the same effect of experimental variables as the latency measures. The product-moment correlation between latency and error computed for the 24 cells in the experiment and averaged over subjects was .42. The 1\% error was obtained in the no-noise condition.

(Manuscript received February 13, 1984; accepted for publication July 12, 1984.) 